\title{
Results of the EUSO-Balloon flight
}

\author{
M. Bertaina* \\ University of Torino and INFN Torino - Italy \\ E-mail: bertaina@to.infn.it
}

P. von Ballmoos

IRAP, Université de Toulouse, CNRS, Toulouse, France

for the JEM-EUSO Collaboration

EUSO-Balloon was launched by the French Space Agency CNES from the Timmins base in Ontario (Canada) on the moonless night of August 25, 2014 UT. After reaching the floating altitude of about $38 \mathrm{~km}$, EUSO-Balloon imaged the UV intensity in the wavelength range 290 $430 \mathrm{~nm}$ for more than 5 hours, before descending to ground, using the key technologies of JEMEUSO. A detailed and precise measurement of the UV intensity in different atmospheric and ground conditions was achieved. The instrument detected hundreds of laser tracks with similar characteristics to Extensive Air Showers. A review of the main physics and technological results obtained by EUSO-Balloon is presented.

35th International Cosmic Ray Conference - ICRC2017

10-20 July, 2017

Bexco, Busan, Korea

\footnotetext{
* Speaker.
} 


\section{Introduction}

The EUSO-Balloon experiment [1] is a pathfinder mission for JEM-EUSO (Extreme Universe Space Observatory on-board the Japanese Experimental Module) [2]. The main objectives of the EUSO-Balloon pathfinder are to perform: a) a full scale end-to-end test of all the key technologies and instrumentation of the JEM-EUSO detector; b) a detailed and precise measurement of the UV intensity in different atmospheric and ground conditions; c) a first measurement of air shower tracks from the edge of space. For its first flight, EUSO-Balloon was launched by the French Space Agency CNES from the Timmins base in Ontario (Canada) on the moonless night of August 25, 2014 UT. After reaching the floating altitude of about $38 \mathrm{~km}$, EUSO-Balloon imaged the UV intensity in the wavelength range 290 - $430 \mathrm{~nm}$ for more than 5 hours before descending to ground. The refractor telescope consisted of two Fresnel lenses and a focal surface filled with multi-anode photomultipliers. The spatial and temporal resolutions of the detector were $\sim 130 \mathrm{~m}$ and $2.5 \mu \mathrm{s}$, respectively. The full field of view in nadir mode was about 11 degrees. The UV data were complemented by Infrared images taken by an infrared camera on board EUSO-Balloon [3]. During 2.5 hours of EUSO-Balloon flight, a helicopter circled under the balloon operating UV flashers [4] and a UV laser [5] to simulate the optical signals from extreme energy cosmic rays, to calibrate the apparatus, and to characterise the optical atmospheric conditions. During flight EUSO-Balloon took more than 2.5 million images that have been analysed to infer different information: study of the performance of the different parts of the detector; response of the detector to the UV flasher and laser events; UV radiance from the Earth atmosphere and ground in different conditions: clear and cloudy atmosphere, grass, forests, lakes, as well as city lights.

\section{The flight and technological aspects}

The flight trajectory of EUSO-Balloon is shown in Fig. 1. During more than five hours of operation at float, a variety of ground covers have been monitored, including different types of soil and vegetation, wetlands, open water, urban and industrial areas to characterise the light intensity in several conditions. During its flight EUSO-Balloon crossed areas characterised by scattered and broken clouds at low altitudes (around $700-800 \mathrm{hPa}$ ) and ice thick clouds at higher altitudes (around $200-300 \mathrm{hPa}$ ). All this variety of conditions turned out to be an ideal case to test the scenes that JEM-EUSO is expected to view during its orbits on the ISS.

The main objective of the first flight was to operate a full scale end-to-end test of all key technologies and instrumentation of JEM-EUSO detectors. A detailed description of the instrument is reported in [1]. Different configurations of the photomultipliers' gain and thresholds on the photon-counting were applied. The acquisition system performed rather well with an integrated data taking of $15,300 \mathrm{~s}$ of the total time at float of about 18,900 s ( $\sim 81 \%$ of time) [6]. The trigger was provided externally by the CPU at a rate of $\sim 20 \mathrm{~Hz}$. 258,592 events were recorded. Each event is composed by 128 frames (Gate Time Units, GTU) of $2.5 \mu$ s each. The total number of GTUs corresponds to an overall integrated time of $\sim 83 \mathrm{~s}$. This acquisition time, distributed uniformly along the flight, allows to get a detailed temporal evolution of the light intensity on the various locations. 


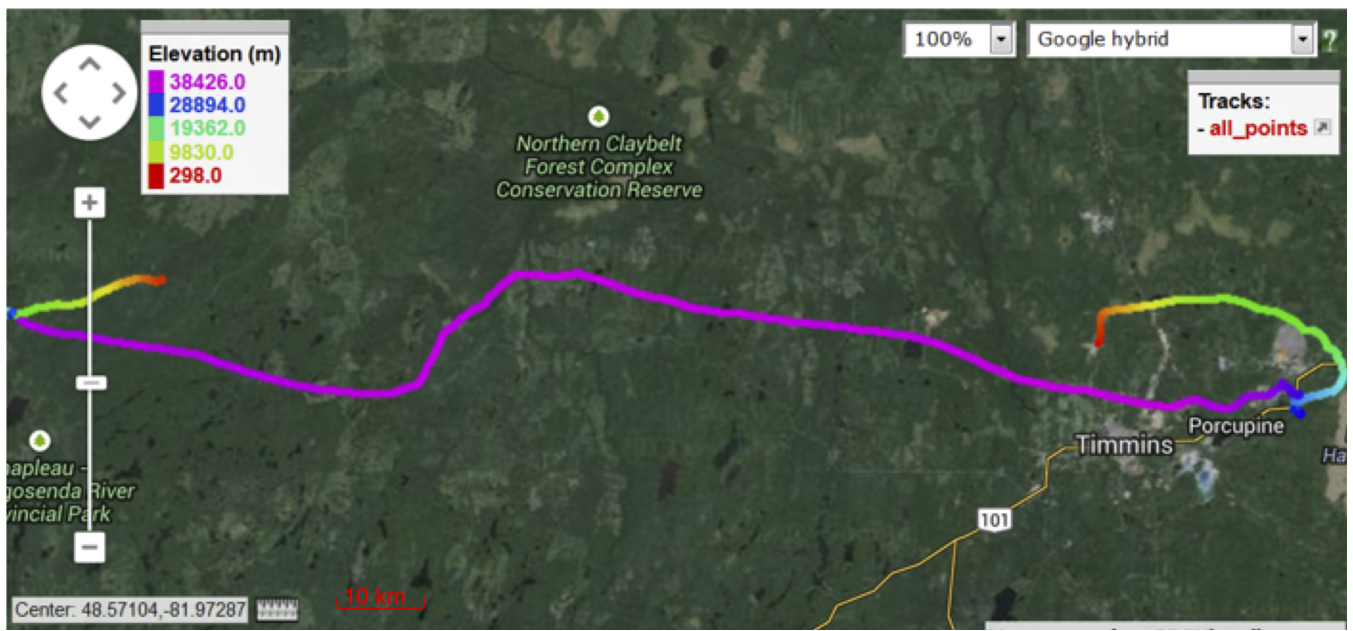

Figure 1: The flight-trajectory of EUSO-Balloon on August 25, 2014 UT. The flight started from the Timmins airport and ended in a tiny lake on the left part of the figure. The elevation of EUSO-Balloon during flight is indicated in the legend. In the first part of the flight EUSO-Balloon imaged mostly urban areas (Porcupine, Timmins, etc.) while the second part of the flight was mostly on forests and lakes.

The front-end electronics and the multi-anode photo-multipliers (MAPMT) behaved rather well. Only 1 over 9 Elementary Units (EC) had a failure. In total only 5 over 36 MAPMTs could not be used for data analysis. A detailed calibration of the 36 multi anode photo-multipliers (MAPMTs) for a total of 2,304 pixels was performed before flight and repeated after recovering the instrument. Details are reported in [7]. Among the active pixels, about $25 \%$ of them were chosen, based on their performance, to get an accurate measurement of the UV light intensity. A detailed modelling of the electronics response has been developed to estimate at best the detector sensitivity and the photon-counting rate, subtracting the electronic noise of the system [8]. The performance of the optics system was carefully studied, being one of the key parameters in a refractive telescope such as JEM-EUSO [9]. The data collected by EUSO-Balloon were used to fine tuned the trigger algorithms which will be employed in future missions [10]. The data collected by EUSO-Balloon were carefully studied [11] in order to estimate the capability of detecting EAS with a much longer flight, such as EUSO-SPB mission [12].

\section{UV light measurements}

The main scientific objective of the EUSO-Balloon flight is the measurement of the UV light intensity. This is relevant for JEM-EUSO as it is one of the key parameters to estimate the exposure curve as a function of energy [13]. Measurements have been performed in the past by BaBy [14], NIGHTGLOW [15] and Sakaki et al. [16]. However, EUSO-Balloon uses a very different approach. It is based on an optical refractive system with very fine spatial and temporal resolutions, the filter has a large bandwith $(290-430 \mathrm{~nm})$ which requires a careful computation of the optics and detector response to translate the detected counts into an absolute measurement. From the point of view of the capability of a space-based observatory for UHECRs the essential point is the the number of counts per GTU at pixel level. This is the pedestal that should be dark enough to 


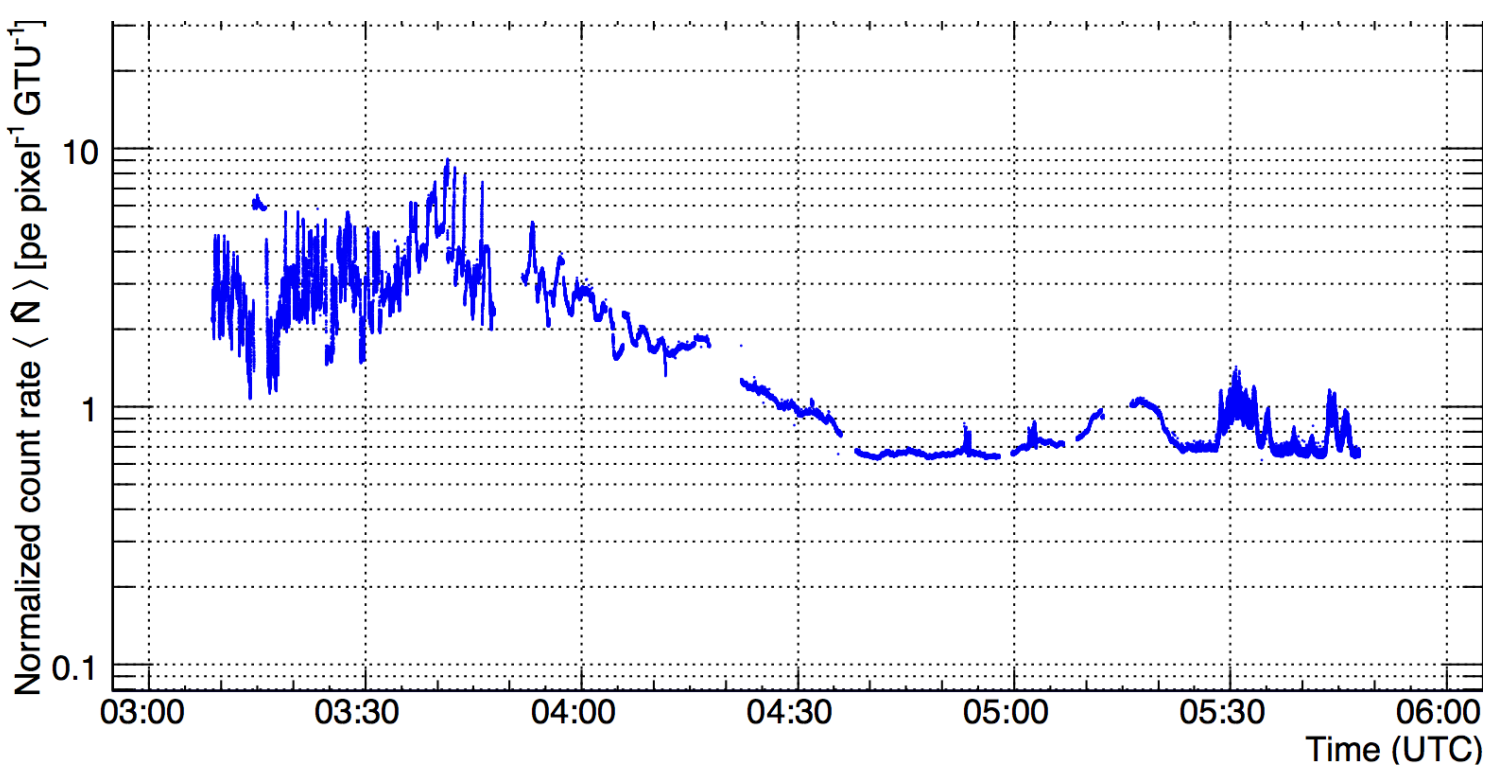

Figure 2: Average normalized count rates $\langle\hat{N}\rangle$ as a function of the packet time. Several breaks are present due to the interruption of measurement necessary to switch between different data acquisition modes.

detect a UHECR track on top. The conversion of the digital counts of the pixels into UV intensity has to take into account many aspects. Among them the most relevant are: entrance aperture of the optics and its throughput, MAPMT detection and filter efficiencies, pixel's field of view and GTU duration. Many of these parameters are wavelength dependent. An accurate determination of all these parameters was performed and is being discussed in [7, 8, 9]. Fig. 2 shows the average normalized count rates $\langle\hat{N}\rangle$ as a function of the packet time. Several breaks are present due to the interruption of measurement necessary to switch between different data acquisition modes which were part of the technological tests foreseen for this flight. As mentioned earlier, it is primarily the diffuse light which is relevant for space-based UHECR observations. The count rate, $\hat{N}_{0}$, for clear atmosphere conditions is an input parameter to the EAS simulations used to estimate the reference aperture for UHECR observations. The corresponding absolute intensity $\left(I_{0}\right)$ is an important factor. This information is useful for the design of any space-based UHECR observatory, independent of the configuration of the instrument.

In EUSO-Balloon, only the back-scattered light from the airglow and extraterrestrial light contributes to the measured diffuse light. The reflectivity of the clouds is higher. Thus, the interval and area with lowest count rates is assumed to represent a case with little influence from clouds, i.e. clear atmosphere. Such conditions were assumed to be present between 04:38 and 04:52. Based on the average of the distribution in that time window, the reference $\hat{N}_{0}$ value is $\sim 0.65$, the FWHM of the pixel distribution being $\sim 0.03$. Between 04:20 and the end of measurement, when the artificial lights of Timmins and surroundings were out of the FoV, the count rate varies within a factor of $\sim 2$. This gives the maximal difference of UV intensity between clear and cloudy sky conditions during flight. Ray trace simulations were then performed using the OffLine code [17] to translate the counts on the pixel in number of photons reaching the pupil of the detector. In the area with no artificial light sources, the diffuse light incident on the EUSO-Balloon telescope is 
purely back-scattered light. We assume two spectrum models based on the spectra incident on the top of the atmosphere and the radiation transfer simulations. The airglow model is deduced from data taken by the Ultraviolet Visual Echelle Spectrograph (UVES) [18, 19]. The starlight model is from Ref. [20]. Under these assumptions, the measured count rate from the diffuse light under clear atmosphere conditions is in the range $I_{0}=300-320$ photons $\mathrm{m}^{-2} \mathrm{sr}^{-1} \mathrm{~ns}^{-1}$ in the $300-500 \mathrm{~nm}$ band. These values do not take into account the systematic uncertainties in the absolute response of the detectors which is currently estimated to be of at least $12 \%$ due to the absolute calibration of the MAPMTs and of the optics response. A more accurate estimation of this value is in progress.

\section{Laser Track Events}

The helicopter events revealed to be extremely useful to understand the system's performance (optics, photo-detector and front-end electronics) and to test the capability of EUSO-Balloon to detect and reconstruct EAS-like events. The EUSO-Balloon configuration has been implemented inside the JEM-EUSO OffLine package [17]. Laser tracks are used to test the reconstruction algorithms [5]. The analysis is based on the geometry of the triggered pixels to constrain the evolution of the event on the so called Shower Detector Plane (SDP). A first guess of the shower geometry is considered, then the difference between the expected and the observed time the signal goes through the pixels is used to further constrain the geometry. In this way, the distance of closest approach, $R_{P}$, and the angle from horizontal to $R_{P}, \Psi_{0}$, are determined to provide information on the shower axis. The typical time fit of a laser event and the direction reconstruction are shown in Fig. 3 after requiring basic quality cuts: a) a set of events of same energy, which reduces uncertainties in the tilting angle of the helicopter, variable on time; b) a track lasting at least 6 GTUs to avoid too short tracks; c) a $\chi^{2} \leq 6$. The figure indicates that $\sim 75 \%$ of the events are located within 1 degree from the median. The outliers are mainly due to incomplete tracks, because only a fraction of the pixels were working at nominal conditions, and to the large spot size which affects the time-position information. Details of the analysis can be found in [5]. It is important to remember that the read-out period of $2.5 \mu$ is optimised for JEM-EUSO, which is expected to detect EAS at $\sim 400 \mathrm{~km}$ distance, instead of $\sim 35 \mathrm{~km}$ as in case of EUSO-Balloon. The fact that EAS-like tracks can be reconstructed also in EUSO-Balloon is quite promising in view of JEM-EUSO.

\section{Unidentified events}

An analysis has been performed to investigate the sensitivity of the instrument to coherent fluctuations of the UV intensity over a large area up to the entire field of view, on timescales of a few to a few tens of $\mu \mathrm{s}$ and search for significant fluctuations [21]. The developed methodology, which is based on the significance to remove the not-uniformity of the detector, allowed to discover, events of different nature: unresolved laser tracks which were not selected by the offline trigger algorithm, presumed artificial light events, stray light events, as well as electronic related events and even unidentified ones. The unidentified event is shown in Fig. 4, where we plot the significance signal for each individual EC as a function of time (left panel), as well as at the level of the whole PDM (right panel). A very clear excess in the photon count rate can be seen around the middle of the data packet, with a significance of $\sim 6 \sigma$ at the PDM level. The signal duration is about 4 

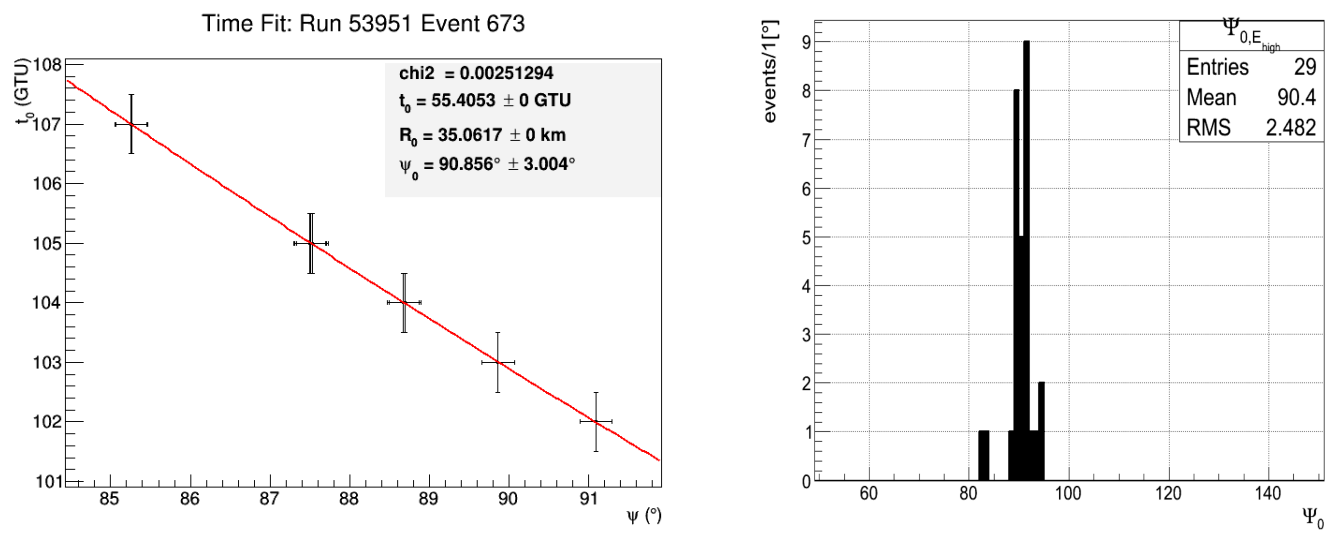

Figure 3: Left: Timing profile of a laser event with constant 0.5 GTU timing error. Right: Zenith angle reconstruction of the helicopter laser shots with the 2 parameter fit method.

GTUs, i.e. $10 \mu \mathrm{s}$. The fact that it can be visible at least in the 6 top ECs suggests on the one hand that the signal is not a mere statistical fluctuation of the data count rate (especially since the significance reaches a level of almost $6 \sigma$ ), and on the other hand that it is indeed not associated with a localised light source in the field-of-view of the instrument. When looking at individual PMTs, it also appears that most of them show an excess in the photon count during that time, which further suggests a diffuse origin. An excess of light over a large fraction of the focal surface could in principle be due to an event within the instrument itself, for instance associated with the interaction of a cosmic ray in the lenses or other parts of the detector. However, the time scale for such an event would be of the order of a GTU, while a detailed analysis in the 10 GTUs around the excess show that the significance takes 3-4 GTUs to cross the entire FoV. Therefore, the event under consideration is most probably related to an actual excess of incoming light into the optical system. Unfortunately, it looks difficult that the event could be associated to a UHECR, assuming that most of the diffuse light comes from reflected Cherenkov light over clouds, because the event should have been of energy well above $10^{19} \mathrm{eV}$. Other speculations include a very short and bright light source located outside the field-of-view of the instrument, e.g. originating from an airplane, whose light could be reflected on the ground.

\section{Conclusions and Perspectives}

The EUSO-Balloon flight in 2014 allowed a full scale end-to-end test of most of the key technologies and instrumentation of JEM-EUSO detectors. EUSO-Balloon was then refurbished for a second flight (EUSO-SPB) of much longer duration [12]. A detailed measurement of the UV intensity in different atmospheric and ground conditions was achieved. The detection of laser events proved the feasibility of the observation of EAS-like events. A detailed analysis of the recorded data allowed to extract events with different signatures: i) stray-light events, ii) "mine" events, and iii) unidentified event which indicate the great variety of signals that could be seen from space and which challenge the observation of UHECRs from space. 

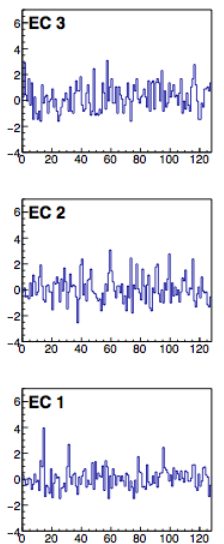
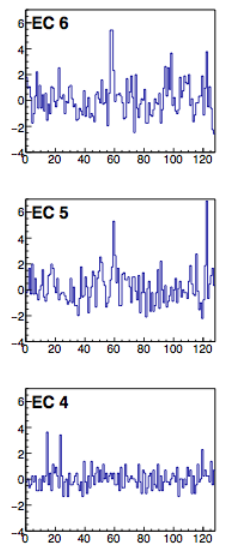
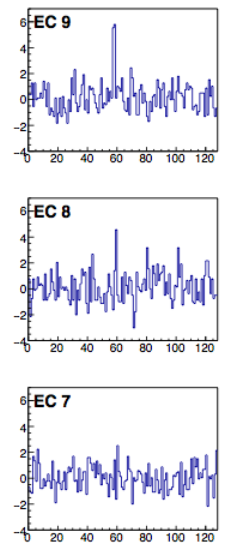

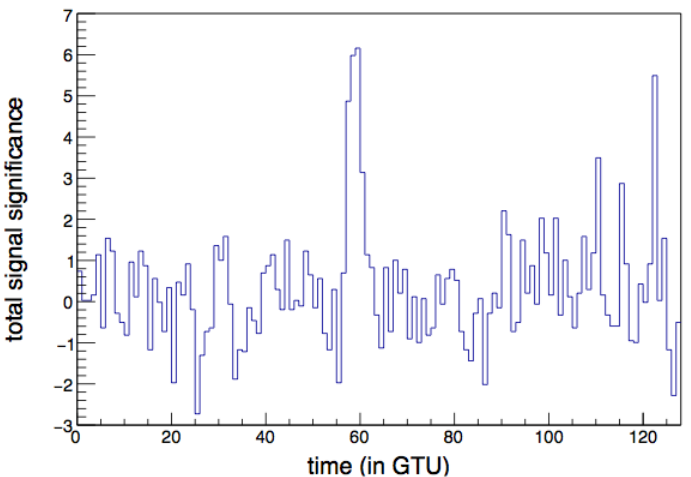

Figure 4: Time sequence showing the photon count significance signal as a function of time (in GTU) in each individual EC (left) and in the whole PDM (right), for the packet in which an unidentified event was recorded.

\section{Acknowledgments}

This work was partially supported by the Basic Science Interdisciplinary Research Projects of RIKEN and JSPS KAKENHI Grant (22340063, 23340081, and 24244042), by the Italian Ministry of Foreign Affairs and International Cooperation, by the 'Helmholtz Alliance for Astroparticle Physics HAP' funded by the Initiative and Networking Fund of the Helmholtz Association, Germany, and by Slovak Academy of Sciences MVTS JEM-EUSO as well as VEGA grant agency project 2/0076/13. Russia is supported by the Russian Foundation for Basic Research Grant No 13-02-12175-ofi-m. The Spanish Consortium involved in the JEM-EUSO Space Mission is funded by MICINN \& MINECO under the Space Program projects: AYA2009-06037-E/AYA, AYA-ESP2010-19082, AYA-ESP2011-29489-C03, AYA-ESP2012-39115-C03, AYA-ESP2013-47816C4, MINECO/FEDER-UNAH13-4E-2741, CSD2009-00064 (Consolider MULTIDARK) and by Comunidad de Madrid (CAM) under projects S2009/ESP-1496 \& S2013/ICE-2822. The authors acknowledge strong support from the French Space Agency CNES who provided - besides funding - the leadership that made the EUSO-Balloon project possible in a very short time. We are deeply indebted to the balloon division of CNES for a perfect launch, smooth flight operation and flawless telemetry. The Canadian Space Agency has provided outstanding facilities at the Timmins Stratospheric Balloon Base, and a quick and careful recovery of the instrument.

\section{References}

[1] J.H. Adams et al. (JEM-EUSO Coll.), Exp. Astronomy 40 (2015) 281.

[2] J.H. Adams et al. (JEM-EUSO Coll.), Exp. Astronomy 40 (2015) 3.

[3] M.D. Rodríguez Frías et al. (JEM-EUSO Coll.), Proc. 34th Int. Cosmic Ray Conf. (Den Haag), \#1309 (2015).

[4] J.H. Adams Jr. et al. (JEM-EUSO Coll.), Proc. 34th Int. Cosmic Ray Conf. (Den Haag), \#0429 (2015). 
[5] J. Eser et al. (JEM-EUSO Coll.), Proc. 34th Int. Cosmic Ray Conf. (Den Haag), \#0860 (2015).

[6] V. Scotti et al. (JEM-EUSO Coll.), Nucl. Instr. \& Meth. A 824 (2016) 655.

[7] C. Moretto, Study of a fluorescence telescope for the EUSO-Balloon project, PhD Thesis, Paris Saclay (2015).

[8] J. A. Rabanal Reina, The EUSO-balloon instrument and an analysis of its photo-detecting efficiency, PhD Thesis, Paris Saclay (2016).

[9] C. Catalano, First light of the EUSO-Balloon telescope: toward the detection of ultra-high energy cosmic rays from space, $\mathrm{PhD}$ Thesis, Toulouse (2015).

[10] G. Abdellaoui et al. (JEM-EUSO Coll.), Nucl. Instr. \& Meth. A 866 (2017) 150.

[11] S. Bacholle, Photo-detection for space-based observation of Ultra-High Energy Cosmic Rays with JEM-EUSO and EUSO-Balloon, PhD Thesis, Paris (2016).

[12] L. Wiencke et al. (JEM-EUSO Coll.), "EUSO-SPB Mission and Science", This Conference Proceedings, Busan (2017).

[13] J.H. Adams Jr. et al. - JEM-EUSO Collaboration, Astroparticle Physics 44 (2013) 76.

[14] S. Giarruso et al., Proc. 28th Int. Cosmic Ray Conf. (Tsukuba), 2 (2003) 849.

[15] L.M. Barbier et al., Astroparticle Physics 22 (2005) 439.

[16] N. Sakaki et al., Proc. 30th Int. Cosmic Ray Conf. (Merida), 5 (2007) 965.

[17] B. Panico et al. (JEM-EUSO Coll.), Proc. 34th Int. Cosmic Ray Conf. (Den Haag), \#1155 (2015).

[18] H. Dekker et al., in Proc. SPIE, Optical and IR Telescope Instrumentation and Detectors, 4008 (2000) 534.

[19] European Southern Observatory; 'UVES-Ultraviolet and Visual Echelle Spectrograph'. http://www.eso.org/sci/facilities/paranal/instruments/uves.html (accessed on August 15, 2016).

[20] C. Leinert et al., Astron. Astrophys. Suppl. Ser. 127 (1998) 1.

[21] A. Jung, JEM-EUSO prototypes for the detection of ultra-high energy cosmic rays (UHECRs): from the electronics of the photo-detection module (PDM) to the operation and data analysis of two pathfinders, PhD Thesis, Paris (2017). 